\title{
CED: Auslassversuch beim Biologikum?
}

\author{
Setzt man bei Patienten mit chronisch-entzündlichen Darmerkrankungen die Biologika ab, \\ erleiden etwa die Hälfte ein Rezidiv. Die erneute Remission ist danach in der Regel möglich.
}

_ TNF-a-Blocker haben die Therapie chronisch-entzündlicher Darmerkrankungen (CED) revolutioniert. Sie bergen allerdings Risiken hinsichtlich unerwünschter Arzneimittelwirkungen und sind zudem sehr teuer. In einer multizentrischen Beobachtungsstudie haben sich spanische Forscher deswegen die Frage gestellt, ob CED-Patienten TNFa-Blocker lebenslang benötigen, oder ob man ein Absetzen riskieren kann. Zudem interessierte sie, ob die Wirksamkeit nach der Therapieunterbrechung erhalten bleibt.

Beobachtet wurden 1.055 Patienten mit Morbus Crohn und Colitis ulcerosa, bei denen unter Infliximab bzw. Adalimumab eine Remission erzielt werden konnte. Die Therapie wurde unterbrochen, und die Patienten wurden zwi- schen 6 und 176 Monate lang nachbeobachtet, im Median über 11 Monate. Insgesamt erlitten 467 von ihnen ein Rezidiv. Daraus konnte man eine kumulative Inzidenz von $24 \%$ nach einem Jahr und $56 \%$ nach fünf Jahren Therapieunterbrechung errechnen. Die mediane Zeit bis zum Rezidiv lag bei elf Monaten.

Von den Patienten mit Rezidiv wurden $69 \%$ erneut mit demselben TNF- $\alpha$ Blocker behandelt. Wenn eine immunsuppressive Therapie weitergelaufen war, konnten bei $78 \%$ dieser Patienten nach dem Wiederansetzen des Biologikums erneut eine Remission erzielt werden. Ohne immunsuppressive Therapie glang dies immerhin bei $62 \%$.

- Casanova MJ et al. Evolution after anti-TNF discontinuation in patients with inflammatory bowel disease: a multicenter long-term follow-up study. Am J Gastroenterol. 2017;112:120-31

\section{KOMMENTAR}

Nach dem Absetzen des Biologikums ist bei der Hälfte der CED-Patienten innerhalb von fünf Jahren mit einem Rezidiv zu rechnen. Allerdings ist das Risiko bei älteren Patienten und solchen, die zusätzlich Azathioprin oder Methotrexat erhalten, deutlich geringer. Bei dieser Konstellation kann man ein Absetzen der Biologikatherapie also eher vertreten. Wurde fortlaufend immunsuppressiv behandelt, konnte bei fast $80 \%$ der Patienten beim Wiederansetzen desselben Biologikums erneut eine Remission erzielt werden. Es ist also nicht zu befürchten, dass sich die Wirksamkeit der Biologika nach einer Therapiepause so sehr abschwächt, dass einem dieser therapeutische Weg verbaut ist.

Prof. Dr. med. H. S. FüeßI

\section{Zarte und stämmige Babys tendieren später zu Fettleibigkeit}

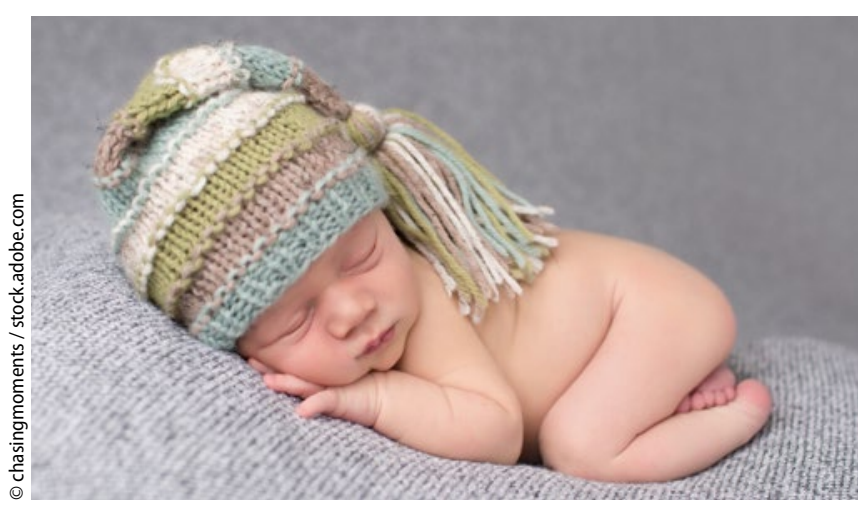

Nur ein normales Geburtsgewicht schützt vor Adipositas.

Zur Frage, inwieweit das Geburtsgewicht das Risiko für eine viszerale Adipositas später im Leben beeinflusst, gab es bisher nur wenige Daten, die über Anekdoten hinausgehen. Eine Studie mit 575 Jugendlichen im Alter von 14-18 Jahren ging dem nun systematisch nach. Das Geburtsgewicht wurde der Patientenakte entnommen. Analysiert wurden Nüchternzucker, Insulin, Lipide, Adiponek- tin, Leptin und CRP, außerdem wurde die viszerale Fettmasse mit Magnetresonanzspektroskopie bestimmt.

In der Datenanalyse ergab sich eine U-förmige Kurve bei der Korrelation von kardiometabolischen Risikofaktoren und Geburtsgewicht. Auch für die Insulinresistenz, den Leptinwert und das viszerale Fett war dieser Zusammenhang signifikant. Der Einfluss von Alter, Geschlecht, Ethnie, Entwicklungsreife, körperlicher Aktivität, sozioökonomischem Status und BMI wurde berücksichtigt.

Interessanterweise ergab sich für Nüchternglukose, Blutdruck, Cholesterin, Adiponektin und CRP sowie die subkutane Fettmasse keine Assoziation mit dem Geburtsgewicht.

Die Studie zeigt, dass zu niedriges, aber auch zu hohes Geburtsgewicht für das Kind zumindest bis zum 18. Lebensjahr einen Nachteil im Hinblick auf Adipositas bedeutet. Interessanterweise besteht der Zusammenhang in erster Linie zur Akkumulation von viszeralem Fett, da hier vermutlich durch embryonales Programming das viszerale Fett als Speicherorgan stärker aktiviert ist.

Prof. Dr. med P. Schwarz

- Stansfield BK, Fain ME, Bhatia J et al. Nonlinear relationship between birth weight and visceral fat in adolescents. J Pediatr. 2016;174:185-92 